REGARDS

SUR LECONOMIE ALLEMANDE

BULLETIN ECONOMIQUE DU CIRAC
Regards sur l'économie allemande

Bulletin économique du CIRAC

$82 \mid 2007$

Varia

\title{
Stihl : la compétitivité d'une société familiale
}

\section{Caroline Guiot}

\section{OpenEdition}

\section{Journals}

Édition électronique

URL : http://journals.openedition.org/rea/641

DOI : $10.4000 /$ rea.641

ISBN : 978-2-8218-0860-7

ISSN : 1965-0787

Éditeur

CIRAC

Édition imprimée

Date de publication : 1 juillet 2007

Pagination : 25-30

ISSN : 1156-8992

Référence électronique

Caroline Guiot, "Stihl : la compétitivité d'une société familiale », Regards sur l'économie allemande [En ligne], 82 l juillet 2007, document 4, mis en ligne le 01 juillet 2009, consulté le 19 avril 2019. URL : http://journals.openedition.org/rea/641; DOI : 10.4000/rea.641 


\section{Stihl : la compétitivité d'une société familiale}

\section{Caroline Guiot}

Le 18 avril 2007, Hans-Peter Stihl a fêté ses 75 ans. S'il fut président de la Fédération des CCl allemandes (DIHT) de 1988 à 2001, réputé pour son rôle actif dans la définition des orientations politiques du monde patronal à l'échelon fédéral, il n'a jamais cessé de piloter l'entreprise familiale du même nom, fondée en 1926 par son père, Andreas Stihl. Sous la direction du fils, l'exigence d'une qualité sans concession des produits et l'internationalisation ont été les facteurs-clefs ayant permis à la petite société Stihl de devenir le leader mondial des tronçonneuses: en quarante ans, le chiffre d'affaires passe de quelque 100 millions $€$ à plus de 2 milliards $€$. Ce succès en fait aujourd'hui une des entreprises-types étudiées dans les enseignements de management stratégique. Elle est en effet l'un de ces hidden champions caractéristiques de l'économie allemande et qui ont patiemment construit leur compétitivité mondiale sur une politique d'innovation basée sur la veille systématique des mutations de leur environnement et centrée sur la qualification des ressources humaines. Restée familiale jusqu'en 2002, la direction de la société a été ensuite réorganisée par Hans Peter Stihl qui avait choisi de ne pas se représenter à la tête de la DIHT pour mieux préparer lui-même sa succession.

En 1926, Andreas Stihl fonde l'entreprise A. STIHL Maschinenfabrik à Stuttgart, dans ce Bade-Wurtemberg réputé pour sa culture industrieuse (voir REA 74/ 2005). Elle compte alors deux salariés et fabrique des foyers de précombustion pour des chaudières à vapeur. La même année, il réalise la première tronçonneuse électrique : elle pèse 48 kilos et doit être maniée par deux hommes. C'est le point de départ d'une série d'innovations destinées à rendre les machines plus sûres, plus légères, plus maniables. Pour preuve, la première tronçonneuse à moteur essence véritablement portable (elle pèse $11 \mathrm{~kg}$ ) est désignée par les initiales BLK : Benzin (essence), Leicht (légère), Klein (pratique et compacte). Mais c'est en 1959 que la Stihl Contra, désormais légendaire, remporte un succès mondial. A. Stihl est dès lors définitivement considéré comme le " père de la tronçonneuse ". Les innovations et les modèles se succèdent. En 1971, le fondateur fête ses 75 ans, et Stihl devient, avec plus de 340000 unités vendues, la marque de tronçonneuses la plus vendue dans le monde, position qui n'a jamais été démentie depuis. En 1992, Stihl acquiert la société autrichienne Viking, élargissant ainsi sa gamme vers les appareils et outils d'entretien des jardins.

Le premier des facteurs-clefs du succès du groupe réside dans l'exigence d'une qualité sans concession des produits. Et la singularité de la production des appareils à moteurs Stihl réside dans le choix assumé d'une intégration verticale de la fabrication : dans un marché globalisé, où une majorité d'entreprises sous-traite une grande partie de leur production ou des pièces détachées pour ne conserver que l'assemblage de leurs produits, Stihl a préféré étendre la maîtrise de la fabrication de ses outils, en l'élargissant aux machines nécessaires à cette production. Pour exemple, le fournisseur de cylindres pour les moteurs des appareils Stihl livrait aussi le secteur automobile du Bade-Wurtemberg. Une surcharge d'activité dans ce secteur entraînait fréquemment des retards d'approvisionnement pour Stihl, considéré comme un client de moindre importance. Qu'à cela ne tienne, Stihl transforme ce point faible en atout : la fabrication desdits cylindres

Création de la tronçonneuse : la réponse à un besoin 
est intégrée dans sa propre chaîne de production en 1996 et celle des pistons des moteurs en 2000. Stihl devient ainsi le seul fabricant de cette industrie à produire non seulement tous les composants de ses moteurs, mais aussi les chaînes et guide-chaînes de ses tronçonneuses. Outre une parfaite adéquation des pièces les unes avec les autres, Stihl améliore significativement la qualité de ses produits, sa flexibilité et sa rentabilité.

La retombée en termes d'image de cette intégration n'est pas négligeable, la réputation mondiale du made in Germany reposant justement sur une garantie de qualité à la fois du produit et des compétences du réseau de distribution. La communication du groupe valorise ces aspects. La compétence des clients, intégrée dans le lien commercial et, plus important encore, dans la conceptiondéveloppement des produits, se trouve en outre mise en avant lors d'événements comme les compétitions mondiales de bûcheronnage : les Timbersports.

\section{STIHL® TIMBERSPORTS $\circledast$ SERIES: “ La force départage, mais la précision l'emporte ! 》}

Les travailleurs forestiers ont de tout temps organisé des rencontres où ils rivalisaient de force et d'habileté afin de déterminer les « meilleurs » de leur profession à l'échelon régional. Cette tradition, ayant ses racines au Canada, en Nouvelle-Zélande et aux Etats-Unis, s'est transformée peu à peu en des compétitions professionnelles d'un haut niveau sportif. En 1985, la société Stihl a créé aux Etats-Unis une série de compétitions de bûcheronnage : les STIHL® TIMBERSPORTS® SERIES. Les premiers championnats d'Europe se sont déroulés en 2002 à Munich, et le premier championnat du monde officiel s'est tenu en septembre 2005 aux Etats-Unis. Les prochains championnats d'Europe ont lieu en août 2007, à Waibligen, siège du groupe. Ils seront retransmis, comme d'habitude, par la chaîne Eurosport. Les compétitions se composent de 6 épreuves.

Standing Block Chop : simulation de l'abattage d'un arbre à la hache. L'objectif est de trancher à la hache, latéralement et le plus rapidement possible, un tronc de $30 \mathrm{~cm}$ de diamètre fixé verticalement.

Underhand Chop : simulation du découpage d'un arbre déjà abattu. Debout sur un tronc de $32 \mathrm{~cm}$ de diamètre ancré horizontalement, les sportifs s'efforcent de le trancher à la hache.

Springboard : deux tremplins sont placés dans un tronc vertical. II s'agit d'abattre une bille de $27 \mathrm{~cm}$ de diamètre placée au sommet. L'épreuve remonte à l'époque où les bûcherons grimpaient dans l'arbre pour abattre les parties hautes du tronc.

STIHL Stock Saw : dans cette épreuve, tous les participants utilisent une tronçonneuse STIHL en vente dans le commerce, la MS 660. Après échauffement, la tronçonneuse est déposée à terre, puis saisie et appliquée le plus rapidement possible sur la bille de $40 \mathrm{~cm}$ de diamètre au moment du coup d'envoi. Deux disques doivent être sciés par un mouvement descendant et un mouvement montant sur une bille horizontale. Les disques doivent avoir l'épaisseur spécifiée, sous peine de disqualification.

Single Buck : un disque est scié à l'aide d'une scie à main (scie «passepartout ») d'environ 2 mètres de long sur une bille horizontale de $46 \mathrm{~cm}$ de diamètre.

Hot Saw : il s'agit de scier le plus rapidement possible trois disques d'épaisseur définie sur une bille horizontale de 46 cm de diamètre. Les concurrents peuvent utiliser soit leur propre tronçonneuse, soit une Hot Saw fournie par Stihl. L'attrait de cette épreuve est qu'il faut maîtriser la puissance de cette tronçonneuse (jusqu'à $62 \mathrm{ch}$ ) d'un poids de $27 \mathrm{~kg}$ et équipée d'une chaîne tournant à $240 \mathrm{~km} / \mathrm{h}$.

Source : www.stihl.de et www.waibligen.de

De l'internationalisation des ventes...

\section{... à l'internationalisation de la production}

Dès 1931, A. Stihl rapporte de Russie et des Etats-Unis des commandes de tronçonneuses à exporter dans ces deux pays. Dès l'année suivante, les premières relations commerciales sont nouées avec une maison italienne, Defries à Milan, qui sera le premier importateur. Pour soutenir le développement des ventes, la question du réseau de distribution se pose. Le choix s'est porté sur un réseau d'importateurs et de revendeurs spécialisés, connaissant parfaitement les produits, régulièrement formés par Stihl, et pouvant assurer un conseil et un service après-vente de qualité auprès des acheteurs et utilisateurs des appareils de la marque. Le lien tissé avec les clients est très fort, reflétant l'exigence de qualité des produits, et les commentaires de ces derniers jouent un rôle important dans l'amélioration constante des produits, voire dans l'élaboration de nouveaux outils. Le réseau de distribution, dont l'étendue s'est accélérée depuis les années 1980 , compte actuellement 32 succursales, plus de 120 importateurs et environ 35000 revendeurs spécialisés répartis dans plus de 160 pays dans le monde.

Parallèlement à l'essor de son réseau de distribution, et afin d'être au plus près de ses différents marchés, Stihl a élargi ses capacités de production, les implantant à l'étranger. Après avoir installé, reconstruit et agrandi ses usines sur le site de Waiblingen (déménagées de Stuttgart après la seconde guerre mondiale), de nouveaux sites ont ouvert leurs portes, soit un total de cinq usines à travers le monde (Suisse, Etats-Unis, Brésil, Chine, et Viking Autriche), en plus de la maison-mère. La société réalise près de $90 \%$ de son CA hors d'Allemagne. 
Groupe Stihl : chiffres clefs en 2006

\begin{tabular}{|lrrrrr|}
\hline & 2004 & 2005 & Evol. & 2006 & Evol. \\
Chiffre d'affaires (en millions $€$ ) & 1621,4 & 1811,9 & $11,7 \%$ & 2018,7 & $11,4 \%$ \\
Part du CA réalisé hors d'Allemagne & $88,0 \%$ & $88,3 \%$ & & $87,9 \%$ & 9449 \\
Nombre d'employés (au 31/12/2006) & 7880 & 8576 & $8,8 \%$ & $10,2 \%$ \\
Investissements (en millions $€$ ) & 114,2 & 137,5 & $20,4 \%$ & 164,2 & $19,4 \%$ \\
\hline
\end{tabular}

Source des données : Geschäftsbericht Stihl Gruppe 2006 ; calculs de l'auteur.

II est à noter que $22 \%$ des investissements réalisés en 2006 ont bénéficié au site de Waiblingen et $78 \%$ aux autres entités en Allemagne et dans le reste du monde. Sur les 164,2 millions € d'investissement, près de $90 \%$ concernent les entités de production. Si l'usine de Qingdao en Chine vient tout juste d'ouvrir ses portes le 15 septembre 2006, il s'agit essentiellement pour les autres sites de moderniser des chaînes de montage, d'agrandir des lieux de stockage des matières premières et des produits finis, ainsi que de multiplier les capacités de production, en ajoutant terrains, nouveaux bâtiments et nouvelles unités de fabrication, pour répondre à une demande grandissante.

Le secret de la réussite de l'entreprise repose donc sur une stratégie de diversification systématique au cœur de laquelle se trouvent le management qualité et l'innovation. Après une phase d'intégration verticale, Stihl a mené une diversification horizontale systématique, élargissant ses activités par-delà le segment de l'outillage professionnel pour investir celui de la demande grand public, tiré par le boom des activités loisirs/nature. L'innovation n'est pas en reste : innovation produit (réduction des émissions de gaz d'échappement, par exemple), mais aussi innovation process. La société a investi 40 millions $€$ dans l'extension de son site de R\&D à Waiblingen et développe une série d'actions de sensibilisation avec les universités et les Fachhochschulen du Land (filières d'ingénieur et technicien), sans négliger la relève féminine au niveau des collèges.

\section{Une culture de responsabilité et d'engagement social}

Dans les coulisses de cette réussite internationale, la direction de la PME Stihl est longtemps restée familiale. Les enfants d'A. Stihl ont rejoint l'entreprise : Eva Mayr-Stihl en 1959 et le fils aîné, Hans Peter Stihl, un an plus tard au poste de directeur adjoint. Lorsque le fondateur décède en 1973, la direction de la société est reprise par ses enfants, associés à hauteur de chacun $25 \%$ du capital : Hans Peter Stihl, Eva Mayr-Stihl, Gerhild Schetter née Stihl, et Rüdiger Stihl. Préférant que les produits de l'entreprise fassent les gros titres plutôt qu'eux-mêmes, ils ont prôné et prônent toujours la plus grande discrétion dans la gestion de la société.

Avec la mort du fondateur s'éteint aussi le paternalisme qui marquait la culture de l'entreprise. "Andreas Stihl, c'était un vrai patriarche », se souvient le tourneur Georg Weinmann qui travailla 44 ans sur le site de Waiblingen et fut, en tant que représentant élu des salariés et membre du comité consultatif d'IG Metall, viceprésident du Conseil de surveillance du groupe jusqu'en 2003 (metall, 9/2004). Non seulement, sous Hans Peter Stihl, «la relation avec les salariés s'améliore », mais l'entreprise se dote d'une charte garantissant sa responsabilité et son engagement vis-à-vis de la société. Ce texte (Grundsätze gesellschaftlicher Verantwortung) en formule ainsi le principe: "Le groupe Stihl, en tant que partie prenante de la société, reconnaît sa responsabilité envers elle dans toutes les régions [du monde] où elle est présente... Les principes de la responsabilité vis-àvis de la société font partie intégrante de la culture interne de l'entreprise Stihl. Tous les salariés sont appelés à veiller au respect de ces principes. Aux partenaires commerciaux et aux sous-traitants, il est demandé d'adopter des principes similaires et de les respecter dans leur politique d'entreprise ».

Ces principes sont déclinés sous la forme d'un code de gouvernance qui condamne toute forme de travail forcé comme le travail des mineurs, et qui accorde
Des investissements axés sur la production...

... et l'innovation

Une direction familiale

Une culture de la responsabilité vis-à-vis de la société

Eva Mayr-Stihl : le choix de la protection de l'environnement 
une place particulière à l'intégration des personnes handicapées de même qu'à la protection de l'environnement. Ces principes s'appliquent au quotidien dans les activités de l'entreprise, mais donnent lieu aussi à une forme plus institutionnalisée. Ainsi, Eva Mayr-Stihl crée en 1986 avec son mari une fondation qui porte aujourd'hui le nom d'Eva Mayr-Stihl Stiftung. Ses missions sont le soutien aux personnes en difficulté, la protection des animaux, ainsi que la recherche, le développement et la formation permettant de supporter ces objectifs. En lien avec les activités du groupe Stihl, les questions environnementales sont depuis plusieurs années au cœur des actions de la fondation.

\section{Les tronçonneuses et la forêt}

Comment résoudre l'apparente contradiction d'un fabricant de tronçonneuses, considérées comme outils de destruction, avec la protection des ressources naturelles en général, et celle de la forêt tropicale en particulier ? La Fondation Eva Mayr-Stihl s'investit dans cette problématique : comprendre les dangers pesant sur les forêts tropicales afin de mieux les prévenir. Contrairement aux idées reçues, le bois de ces forêts est en effet extrêmement peu exploité pour le commerce ; il est essentiellement brûlé pour dégager des surfaces cultivables. Or les terres de brûlis sont appauvries et accélèrent l'érosion des sols. Loin de cette vision de court terme, l'engagement de Stihl est de démontrer qu'une exploitation raisonnée des forêts tropicales participe à la mise en œuvre d'un système économique pérenne pour les populations locales, et à la protection durable des ressources naturelles. La Fondation soutient ainsi des projets de créations de réserves et de pépinières, et apporte son soutien financier à des institutions comme l'International Union of Forest Research Organizations (IUFRO) ou la Tropical Forest Foundation (TPP).

Source : www.stihl.fr et www.iufro.org

Hans Peter Stihl : engagement dans l'économie locale...

... dans la politique partenariale de sa branche...
Hans Peter Stihl, de son côté, s'implique très activement dans la politique de développement régional menée par la $\mathrm{CCl}$ de la région de Stuttgart, dont il a assuré la présidence de 1989 à 2001, et dont il est encore aujourd'hui président d'honneur. Parallèlement, il préside la $\mathrm{CCl}$ du district de Rems-Murr où se trouve Waiblingen, est membre du conseil d'administration de l'antenne locale de l'Agence pour l'emploi de Nuremberg (1974/85), et du directoire de la caisse d'assurance maladie AOK (1970/80). En 2001, il se voit décerner le prix Regionaut qui récompense son engagement au sein du Forum Region Stuttgart, une association à but non lucratif rassemblant un réseau de personnalités de tous horizons et de toute obédience (milieux sportifs, universitaires, politiques, culturels, économiques, syndicaux, culturels, ecclésiastiques, etc.) et qui s'est donné pour mission de "soutenir les forces d'innovation présentes dans la région de Stuttgart " comme de promouvoir la coopération des acteurs régionaux et d'intensifier les réseaux d'échanges avec d'autres régions. Dans ce contexte, H. P. Stihl s'était montré notamment l'un des plus ardents défenseurs de l'extension de l'aéroport de Stuttgart et du site des foires-expositions, projets vitaux pour l'économie de la région. Comme pour tous les patrons allemands, son engagement dans le « capitalisme rhénan " se traduit aussi par sa qualité de membre dans les conseils de surveillance d'autres sociétés: IBM Deutschland GmbH (1988-98), Robert Bosch GmbH (1998-2006), Südwestbank AG in Stuttgart (1978-95) ou IKB Deutsche Industriebank, Düsseldorf (1987-2002).

Son engagement dans la vie régionale l'amène, de 1973 à 1988, à assumer les responsabilités de membre du directoire, puis de président, de la fédération patronale des industries de la métallurgie du Bade-Wurtemberg (Verband der Metallindustrie, VMI). Ce rôle est stratégique pour son entreprise comme pour l'ensemble de la branche, puisque c'est traditionnellement dans ce Land que sont conclus les accords salariaux de branche avec le partenaire syndical IG Metall, en l'occurrence sa puissante direction régionale. C'est ainsi que H.P. Stihl, en qualité de président de la délégation patronale, est amené à négocier en 1984 l'introduction progressive de la semaine de 35 heures dans la métallurgie. Pourtant, H.P. Stihl était un farouche opposant de cette réduction du temps de travail, estimant qu'elle est dommageable à l'emploi et la compétitivité ; mais la VMI a fini par céder après une série historique de grèves dans le Land, affectant au printemps 1984 toutes les entreprises de renom du Land, dont Stihl. Quelque vingt ans plus tard, un accord-pilote conclu dans le Bade-Wurtemberg (2002) et l'accord de Pforzheim (2004) qui permettent aux entreprises de déroger aux minima de branche pour des impératifs de compétitivité, puis la série d'accords conclus à 
l'été 2004 dans un certain nombre de grandes entreprises allemandes (dont Bosch) sur le retour aux 40 heures hebdomadaires (voir REA 68/04) lui donnent raison a posteriori.

Un engagement responsable qui repose sur le principe : « propriété oblige »

Bien entendu, la société Stihl applique ces accords, voyant varier au fil des ans la durée du temps de travail. Elle est en effet liée par les accords de branche, mais aussi par son code de déontologie qui stipule expressément: "Stihl reconnaît la liberté d'association de ses salariés ». Une assertion à première vue inutile, puisque les contrats conclus entre les partenaires sociaux ont force de loi et que toute entreprise allemande est soumise au régime de la cogestion.

En réalité, elle réaffirme un credo fondé doublement sur les valeurs personnelles (protestantes) de H.P. Stihl et sur les valeurs au cœur de l'ordo-libéralisme allemand et qui, au fil de la jurisprudence du Tribunal constitutionnel fédéral, ont pris force de principes constitutionnels (voir REA 52-53/01) : l'équité de droits (et de devoirs) entre travail et capital. Appliquées à l'entreprise - a fortiori si elle est familiale -, elles instituent une coopération de fait, établie sur le principe constitutionnel : "propriété oblige » (art. 14, 2 de la Loi fondamentale).

II signifie que, en usant de son bien à des fins industrielles et commerciales, le propriétaire engage sa responsabilité vis-à-vis de la collectivité puisque, en créant une entreprise, il devient entrepreneur et contracte donc avec la société civile. Patron et salariés constituent une communauté qui crée des richesses, ouvrant droit au partage de ses fruits. Les deux parties prenantes sont liées par la co-responsabilité : sans capital pas de travail (responsabilité du patron) - sans travail pas de capital (responsabilité du salarié).

Cette doctrine au cœur du modèle rhénan est quelque peu mise à mal actuellement avec la montée en puissance d'un troisième acteur : l'actionnaire. C'est là une autre raison expliquant l'affirmation de ces valeurs par une société comme Stihl.

Le rôle et l'influence de H.-P. Stihl dépassent en réalité de loin le cadre local et régional. S'il préside la VMI, il est également vice-président de Gesamtmetall, l'organisation centrale partenaire d'IG Metall à l'échelon fédéral. II assume de même les fonctions de vice-président de l'Institut der Deutschen Wirtschaft proche du BDI (1983/98), de membre du directoire de la Fédération de la construction mécanique (VDMA) et de président de la Fédération des CCl allemandes (DIHT) de 1988 à 2001. Or si H.P. Stihl exerce toutes ces missions au sein de la « triade décisionnelle » où se décide conjointement la politique fédérale, ce n'est pas par appétit du pouvoir ni pour en tirer un quelconque profit au bénéfice de son entreprise. C'est par conviction personnelle. Car, quelle que soit sa position, il défend la doctrine de l'économie sociale de marché; elle figure d'ailleurs en tête des principes éthiques de l'entreprise : «Stihl affirme son attachement au principe de l'économie sociale de marché et de la concurrence ». Cette doctrine repose ellemême sur des principes et valeurs, dont la liberté et, plus encore, la responsabilité individuelle - manifestation première du principe de subsidiarité -, impliquant l'auto-régulation (et pour les collectivités publiques : l'auto-administration, le credo corollaire de H. P. Stihl à la tête des $\mathrm{CCl}$ ). Dans ces fonctions d'envergure nationale, ce patron d'une PME devenue un hidden champion a largement contribué au retour à l'orthodoxie ordo-libérale de la politique menée par le second gouvernement Schröder que prolonge le gouvernement Merkel.

Concrètement, au niveau de la société Stihl, cet attachement aux valeurs se traduit par une culture forte qui mise sur la participation des salariés et engage la responsabilité du patron sur le long terme (c'est d'ailleurs la raison pour laquelle H.-P. Stihl s'occupe lui-même de sa succession). Elle se reflète dans l'importance accordée à la formation des apprentis: le centre de formation Stihl ouvre ses portes dès 1940, et un partenariat étroit est mis en place lors de la création de l'académie des métiers de Stuttgart (Berufsakademie). Le 15 juillet 2005, un nouveau centre de formation a été inauguré, représentant un investissement de 1 million $€$. En 2006, avec 268 apprentis en formation dans ses ateliers, soit environ $7 \%$ de l'effectif de la maison-mère, Andreas Stihl AG \& Co. KG, le groupe mise sur le développement des talents, avec une quote-part d'apprentis supérieure à la moyenne de la branche. Les salariés sont totalement investis dans l'entreprise. Ainsi, le système de recueil des suggestions proposées par le personnel des usines allemandes, mis en place en 1948 (betriebliches Vorschlagswesen), se poursuit encore aujourd'hui sous la forme d'une boîte à idées. Sur 6000 idées recueillies en 2006, près de $63 \%$ ont été mises en œuvre. Parallèlement, plus de 200 groupes de travail sont régulièrement réunis et formés dans le cadre du programme «process d'amélioration continue » (kontinuierlicher Verbesserungsprozess) afin d'améliorer les conditions de travail. Cette implication de chacun dans
... et dans la négociation de la politique économique et sociale à l'échelon fédéral

Responsabilité et engagement fondent la culture de l'entreprise... 
... et incitent son patron à gérer en personne sa succession

Un passage de relais en deux temps la vie de l'entreprise, comme celle de la direction dans la prise en compte des remarques et leur mise en application, se reflète dans la réponse aux questionnaires internes : celui de 2006 montre que $98 \%$ des salariés sont fiers "d'appartenir » à Stihl. Cette politique se traduit également par le développement de l'actionnariat salarié dès 1985, une manière de concilier objectifs sociaux et augmentation du capital, et par des salaires supérieurs aux normes de branche.

La même approche de la responsabilité amène $H$.-P. Stihl à ne pas se représenter à la tête de la DIHT pour préparer l'avenir de l'entreprise familiale. II initie deux transformations importantes dans la structure du groupe : la première concerne la création au $1^{\mathrm{er}}$ janvier 1995 de la Stihl Holding AG \& Co. KG, regroupant les sociétés Andreas Stihl AG \& Co. KG (Waiblingen), les filiales Stihl Vertriebszentrale AG \& Co. KG (Dieburg) et Stihl \& Co (Wil, Suisse); la seconde, en novembre 1997 : les actionnaires de la holding créent la société par actions Stihl AG (Waiblingen) gérée par conseil de surveillance et par un directoire.

En octobre 2001, le groupe annonce le prochain retrait des membres de la famille de la gestion opérationnelle de l'entreprise, à l'occasion du $70^{\mathrm{e}}$ anniversaire du fils du fondateur. Hans Peter Stihl sera dorénavant président du conseil de surveillance de Stihl AG, auquel siègeront également Eva Mayr-Stihl et Rüdiger Stihl. Les trois frères et sœur dirigeront également le comité de direction de la Stihl Holding AG \& Co. KG. Au $1^{\text {er }}$ juillet 2002, c'est, pour la première fois dans l'histoire du groupe, une personne extérieure à la famille Stihl qui prend les commandes: Harald Joos, un transfuge de Schindler. Cette nomination ne conviendra pas, et un an plus tard, il est remplacé par Bertram Kandziora, ancien directeur de production chez Bosch et Siemens. II est cosignataire, avec H.P. Stihl, de la charte de déontologie interne et poursuit le développement de la société dans l'esprit du fondateur.

COMME NOMBRE DE PME ALLEMANDES, LE GROUPE FAMILIAL STIHL a été confronté à la problématique de la succession de la direction de l'entreprise. L'anticipation de cette échéance, ainsi que la fidélité du nouveau président aux principes qui ont assurés le succès du développement de l'entreprise, exigence de qualité et internationalisation, sont les facteurs-clefs qui permettent au groupe de poursuivre son essor, malgré le retrait des membres de la famille fondatrice et de se positionner en "gagnant de la mondialisation ».

Paradoxalement, la culture développée par H.-P. Stihl, un patron plus engagé que les autres, mais représentatif de cette approche de la responsabilité qui caractérise le Mittelstand industriel allemand avec ses structures à dominante familiale, si elle a pu prêter à sourire durant les années où la mode était à la new economy, reprend aujourd'hui, à l'aube de la société du savoir, une importance stratégique. Car en valorisant le facteur humain, la responsabilité et la compétence de chacun au sein d'une culture partenariale ouverte au dialogue et à la coopération, elle permet de préserver une logique interne de compétitivité adaptée à un environnement globalisé. Les valeurs qui la fondent, dont cette approche du développement durable que mettent en œuvre les entreprises d'origine familiale, se révèlent un facteur de compétitivité d'autant plus solide qu'il repose sur une vision évolutive et partenariale de l'environnement. Seul un mode de gouvernance accordant une plus large place aux actionnaires risquerait de menacer cet équilibre des forces entre intérêts particuliers et collectifs qui caractérise, pardelà l'exemple de la société Stihl, la majeure partie du tissu des entreprises familiales allemandes.

\section{Indications bibliographiques}

BOURGEOIS I., «Bade-Wurtemberg : une compétitivité à taille humaine ", Regards sur l'économie allemande, $\mathrm{n} 74 / 2005$

«Stihl : Am liebsten alles selbst machen », Handelsblatt, 26-06-2006

"Familie Stihl-Mayr : die leisen Krachmacher ", manager-magazin.de, 08-04-2004

www.stihl.de ; www.dihk.de ; www.iufro.org ; www.waiblingen.de. 